\title{
The High-Resolution Wave-Propagation Method Applied to Meso- and Micro-scale Flows
}

\author{
Nash'at N. Ahmad*, Fred H. Proctor ${ }^{\dagger}$ \\ NASA Langley Research Center, Hampton, Virginia, 23681
}

\begin{abstract}
The high-resolution wave-propagation method for computing the nonhydrostatic atmospheric flows on meso- and micro-scales is described. The design and implementation of the Riemann solver used for computing the Godunov fluxes is discussed in detail. The method uses a flux-based wave decomposition in which the flux differences are written directly as the linear combination of the right eigenvectors of the hyperbolic system. The two advantages of the technique are: 1) the need for an explicit definition of the Roe matrix is eliminated and, 2) the inclusion of source term due to gravity does not result in discretization errors. The resulting flow solver is conservative and able to resolve regions of large gradients without introducing dispersion errors. The methodology is validated against exact analytical solutions and benchmark cases for non-hydrostatic atmospheric flows.
\end{abstract}

\section{Introduction}

$\mathrm{T}$ he high-resolution wave-propagation method was introduced by LeVeque as a general algorithm for solving hyperbolic conservation laws (LeVeque 1996; LeVeque 1997; LeVeque 2002). It has been successfully applied to a wide range of physical problems (LeVeque 2002). Traditionally, atmospheric modelers have favored the centered finite difference schemes. These types of schemes exhibit non-physical spurious oscillations, which can contaminate the numerical results and introduce false negatives in microphysical scalars. At smaller spatial and temporal scales, large gradients of velocities and other physical quantities can develop, which create stability problems for such schemes. The high-resolution wave-propagation methods are conservative finite volume discretizations and have the ability to resolve regions of steep gradients accurately, thus avoiding dispersion errors in the solution. Positivity of scalars (an important factor when considering the transport of microphysical quantities) is also guaranteed by applying the total variation diminishing (TVD) condition appropriately.

The conservation laws used in atmospheric models are simplified by assuming that the atmospheric processes are adiabatic - the conservation of total energy is replaced by the conservation of potential temperature (Das 1979). Ooyama (1990) stated that "The first law of thermodynamics is a statement of conservation in the processes that involve more forms of energy than the purely mechanical. Since atmospheric processes are predominantly adiabatic, it is appropriate to express the first law in terms of entropy conservation." This has often been the argument for using the potential temperature conservation equation rather than the total energy. Some examples of the flow model with the potential temperature conservation can be found in Proctor (1987) and Bacon et al. (2000). The operational mesoscale models such as the Regional Atmospheric Modeling System (RAMS) developed by Pielke et al. (1993) and the Coupled Ocean/Atmosphere Mesoscale Prediction System (COAMPS) developed by Hodur (1997) are all based on equations which express the conservation of total energy in terms of functions for potential temperature. The conservation laws with total energy equation are rarely used for atmospheric flow computations. Some exceptions include, Botta et al. (2004), Ahmad et al. (2007) and Giraldo and Restelli (2008).

In this study, the high-resolution wave-propagation method is applied to meso- and micro-scale atmospheric flows. The equation set comprises of conservation of total energy rather than potential temperature. There are two major advantages (Giraldo and Restelli 2008) in using the conservation of total energy equation: 1) energy is conserved to machine precision and, 2) true viscous stresses are accounted for if the diffusion term is included. In the following sections the governing equations and the numerical technique are described. The results of benchmark simulations performed to evaluate the accuracy of the numerical scheme and its applicability to nonhydrostatic atmospheric flows are discussed in detail.

\footnotetext{
${ }^{*}$ Research Aerospace Engineer, Crew Systems \& Aviation Operations Branch, Mail Stop 152, AIAA Senior Member.

$\dagger$ Senior Research Scientist, Crew Systems \& Aviation Operations Branch, Mail Stop 152, AIAA Senior Member.
} 


\section{Governing Equations}

The basic equations governing fluid flow comprise of a set of partial differential equations for the conservation of mass, momentum and energy. In two dimensions they can be written as:

$$
\frac{\partial U}{\partial t}+\frac{\partial F}{\partial x}+\frac{\partial H}{\partial z}=\frac{\partial F_{v}}{\partial x}+\frac{\partial H_{v}}{\partial z}+\psi+S
$$

where,

$$
U=\left[\begin{array}{c}
\rho \\
\rho u \\
\rho w \\
E
\end{array}\right], \quad F=\left[\begin{array}{c}
\rho u \\
\rho u^{2}+p \\
\rho u w \\
u(E+p)
\end{array}\right], \quad H=\left[\begin{array}{c}
\rho w \\
\rho u w \\
\rho w^{2}+p \\
w(E+p)
\end{array}\right], \quad \psi=\left[\begin{array}{c}
0 \\
0 \\
\rho g \\
\rho w g
\end{array}\right]
$$

$\rho$ is the density of fluid, $u$ is the velocity component in the $x$-direction, $w$ is the velocity component in the $z$-direction and $p$ is the pressure.

$E$ is the total energy per unit volume:

$$
E=\rho e+\rho \frac{1}{2}\left(u^{2}+w^{2}\right)
$$

where, $\frac{1}{2}\left(u^{2}+w^{2}\right)$ is the specific kinetic energy. The specific internal energy, $e$ is given by an equation of state:

$$
e=\frac{p}{(\gamma-1) \rho}
$$

In the above relations, $\gamma$ is the ratio of specific heats and $g$ is the acceleration due to gravity. In this study the atmosphere is assumed to be dry and the source term $S$ is set to zero ( $S$ would represent heat sinks and sources due to microphysical processes.). The viscous fluxes, $F_{\mathrm{v}}$ and $H_{\mathrm{v}}$ are given by:

$$
F_{v}=\left[\begin{array}{c}
0 \\
\tau_{x x} \\
\tau_{x z} \\
u \tau_{x x}+w \tau_{x z}-\frac{\mu c_{p}}{\operatorname{Pr}} \frac{\partial T}{\partial x}
\end{array}\right], \quad H_{v}=\left[\begin{array}{c}
0 \\
\tau_{x z} \\
\tau_{z z} \\
u \tau_{x z}+w \tau_{z z}-\frac{\mu c_{p}}{\operatorname{Pr}} \frac{\partial T}{\partial z}
\end{array}\right]
$$

In Eq. (5) $\mu$ is the dynamic viscosity, $T$ is temperature, $c_{\mathrm{p}}$ is the specific heat at constant pressure and $\operatorname{Pr}$ is the Prandtl number.

The viscous shear stress tensor, $\tau_{\mathrm{ij}}$ is given by:

$$
\left[\begin{array}{c}
\tau_{x x} \\
\tau_{z z} \\
\tau_{x z}
\end{array}\right]=\left[\begin{array}{c}
2 \mu \frac{\partial u}{\partial x}-\frac{2}{3} \mu\left(\frac{\partial u}{\partial x}+\frac{\partial w}{\partial z}\right) \\
2 \mu \frac{\partial w}{\partial z}-\frac{2}{3} \mu\left(\frac{\partial u}{\partial x}+\frac{\partial w}{\partial z}\right) \\
\mu\left(\frac{\partial u}{\partial x}+\frac{\partial w}{\partial z}\right)
\end{array}\right]
$$




\section{Numerical Scheme}

The conservation laws in Eq. (1)-(2) without the source terms can be written in the discrete form in 1D as:

$$
U_{i}^{n+1}=U_{i}^{n}-\Delta t\left[\frac{1}{\Delta x}\left(F_{i+\frac{1}{2}}-F_{i-\frac{1}{2}}\right)\right],
$$

where $U$ is the vector of conserved quantities, and $F$ is the vector of inter-cell fluxes calculated at the control surface of each control volume using either an exact or approximate Riemann solver. $\Delta t$ and $\Delta x$ are the time step and mesh resolution in $x$-direction, respectively. LeVeque (2002) and Bale et al. (2002) suggest using a flux-based wave decomposition, in which the flux differences $F_{i}\left(U_{i}\right)-F_{i-1}\left(U_{i-1}\right)$ are written directly as a linear combination of the right eigenvectors, $r_{i-1 / 2}^{p}$ :

$$
F_{i}\left(U_{i}\right)-F_{i-1}\left(U_{i-1}\right)-\Delta x \psi_{i-1 / 2}=\sum_{p=1}^{m} \beta_{i-1 / 2}^{p} r_{i-1 / 2}^{p} \equiv \sum_{p=1}^{m} Z_{i-1 / 2}^{p}
$$

where,

$$
\beta_{i-1 / 2}=R_{i-1 / 2}^{-1}\left(F_{i}\left(U_{i}\right)-F_{i-1}\left(U_{i-1}\right)-\Delta x \psi_{i-1 / 2}\right)
$$

The vectors $Z^{p}=\beta^{p} r^{p}$ are called $f$-waves and contain flux increments rather than increments in $U . R_{i-1 / 2}$ is the matrix of right eigenvectors. $\psi=\rho g$, is the source term due to gravity. Eq. (7) can now be re-written as:

$$
U_{i}^{n+1}=U_{i}^{n}-\frac{\Delta t}{\Delta x}\left[\mathrm{H}^{+} \Delta U_{i-1 / 2}+\mathrm{H}^{-} \Delta U_{i+1 / 2}\right]
$$

where,

$$
\mathrm{H}^{-} \Delta U_{i-1 / 2}=\sum_{p} Z_{i-1 / 2}^{p} \quad \text { if } \quad s_{i-1 / 2}^{p}<0
$$

and,

$$
\mathrm{H}^{+} \Delta U_{i-1 / 2}=\sum_{p} Z_{i-1 / 2}^{p} \quad \text { if } \quad s_{i-1 / 2}^{p}>0
$$

The fluctuations $\mathrm{H}^{-} \Delta U_{i-1 / 2}$ and $\mathrm{H}^{+} \Delta U_{i-1 / 2}$ contribute to the cell-averaged quantity $U_{i}$ due to the wave propagation across the cell interfaces. In the above relations, $S_{i-1 / 2}^{p}$ are the wave speeds given by the eigenvalues of the hyperbolic equation set. Higher-order accuracy in space can be achieved by adding a correction term (LeVeque 1996: LeVeque 1997; LeVeque 2002):

$$
U_{i}^{n+1}=U_{i}^{n}-\frac{\Delta t}{\Delta x}\left[\mathrm{H}^{+} \Delta U_{i-1 / 2}+\mathrm{H}^{-} \Delta U_{i+1 / 2}\right]-\frac{\Delta t}{\Delta x}\left[\widetilde{F}_{i+1 / 2}-\widetilde{F}_{i-1 / 2}\right]
$$

where,

$$
\widetilde{F}_{1-1 / 2}=\frac{1}{2} \sum_{p=1}^{m} \operatorname{sgn}\left(s_{i-1 / 2}^{p}\right)\left[1-\frac{\Delta t}{\Delta x}\left|s_{i-1 / 2}^{p}\right|\right] \widetilde{Z}^{p}{ }_{i-1 / 2}
$$


and, $\widetilde{Z}^{p}$ is the limited value of $Z^{p}$. Given the $f$-waves and the wave speeds, the flux differences can be computed by summing up the left and right going waves across a cell interface. In the above relations, the sweep in $x$-direction is implied. Similar methodology can be used for computations in the z-direction with the addition of a source term in the $z$-direction. The quantities on cell faces are calculated by taking the average of cell-centered quantities on the either side of the face.

\section{Results}

In this section the numerical scheme is evaluated against several benchmark cases - (1) isentropic vortex evolution (Yee at al. 1999); (2) transport of density distribution (Deiterding 2000); (3) Rayleigh-Taylor instability (Almgren 2010); (4) inertia-gravity waves on non-hydrostatic scale (Skamarock and Klemp 1994); and (5) density current (Straka et al. 1993). In the first four cases the Euler equations are computed and in the last test case the Navier-Stokes equations are computed. The first two test cases have exact analytical solutions.

\section{A. Isentropic Vortex}

The evolution of a stationary and a moving inviscid isentropic vortex (Yee et al. 1999) is described in this section. In this case, there is no source term due to gravity. The computational domain was defined as $(x, z) \in[0,10] \times[-5,5] \mathrm{m}$ with $t \in[0,100] \mathrm{s}$. The mesh size was set to $100 \times 100$ cells. Periodic boundary conditions were used in all directions for the moving vortex and farfield conditions were used for the stationary vortex. For the stationary vortex, the $u$-velocity and $w$-velocity were set to zero and in the case of the moving vortex the $u$-velocity was set to $0.2 \mathrm{~m} / \mathrm{s}$. The initial density and pressure were set to $1 \mathrm{~kg} / \mathrm{m}^{3}$ and $1 P a$ respectively. Perturbations in the flow field $(\delta u, \delta w)$ and density $(\delta \rho)$ were added such that $\delta S=0$, where, $S$ is the entropy (Yee at al. 1999):

$$
(\delta u, \delta w)=\frac{\beta}{2 \pi} e^{\left(1-r^{2}\right) / 2}(-\bar{z}, \bar{x})
$$

The initial density field was defined by:

$$
\rho=\left[1-\frac{(\gamma-1) \beta^{2}}{8 \gamma \pi^{2}} e^{1-r^{2}}\right]^{1 /(\gamma-1)}
$$

In the above equations $\beta$ is the vortex strength which was set to 5. $r^{2}=\bar{x}^{2}+\bar{z}^{2}$ and $(\bar{x}, \bar{z})=\left(x-x_{0}, z-z_{0}\right)$. The initial vortex location is given by $\left(x_{0}, z_{0}\right)=(5,0)$. The pressure was initialized as follows:

$$
p=\rho^{\gamma}
$$

The root mean square errors in density $\left(E_{\rho}\right)$ for the stationary vortex case were calculated for different mesh resolutions and are listed in Table 1. The computed density fields and the comparison with exact solutions along the mesh centerline are shown in Figure 1 (stationary vortex) and Figure 2 (moving vortex), respectively. The computed solutions are in good agreement with the exact solution and exhibit little numerical diffusion. The solution on a higher-resolution mesh (200 x 200 cells) and a comparison with the exact solution along the mesh centerline is shown in Figure 3.

Table 1: Stationary Isentropic Vortex

\begin{tabular}{|c|c|c|}
\hline \# of cells & $\Delta \mathbf{x}(\mathbf{m})$ & $\boldsymbol{E}_{\boldsymbol{\rho}}$ \\
\hline $50 \times 50$ & 0.2 & $9.41 \mathrm{E}-03$ \\
\hline $100 \times 100$ & 0.1 & $1.34 \mathrm{E}-03$ \\
\hline $200 \times 200$ & 0.05 & $1.82 \mathrm{E}-04$ \\
\hline
\end{tabular}



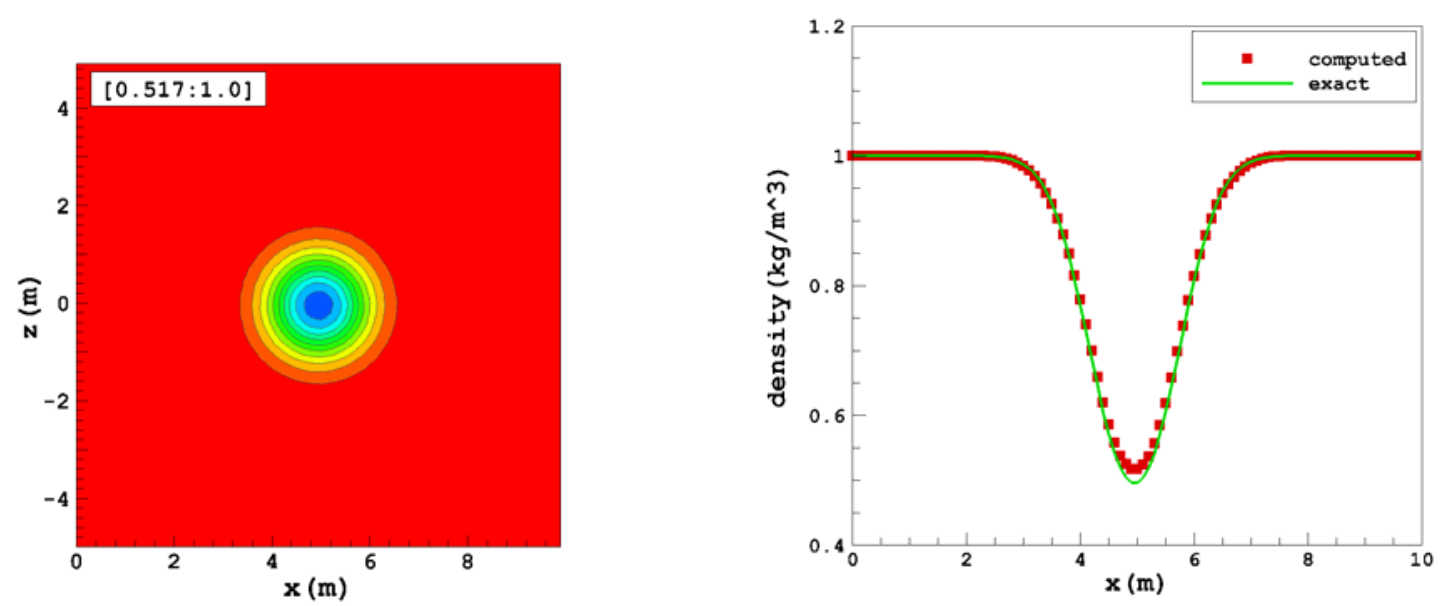

Figure 1. Stationary Vortex. Computed density $\left(\mathrm{kg} / \mathrm{m}^{3}\right)$ at time $=100 \mathrm{~s}$ (left) and the comparison of the computed solution with the analytical solution at $z=0$ and time $=100$ s (right). Mesh size $=100 \times 100$ cells.
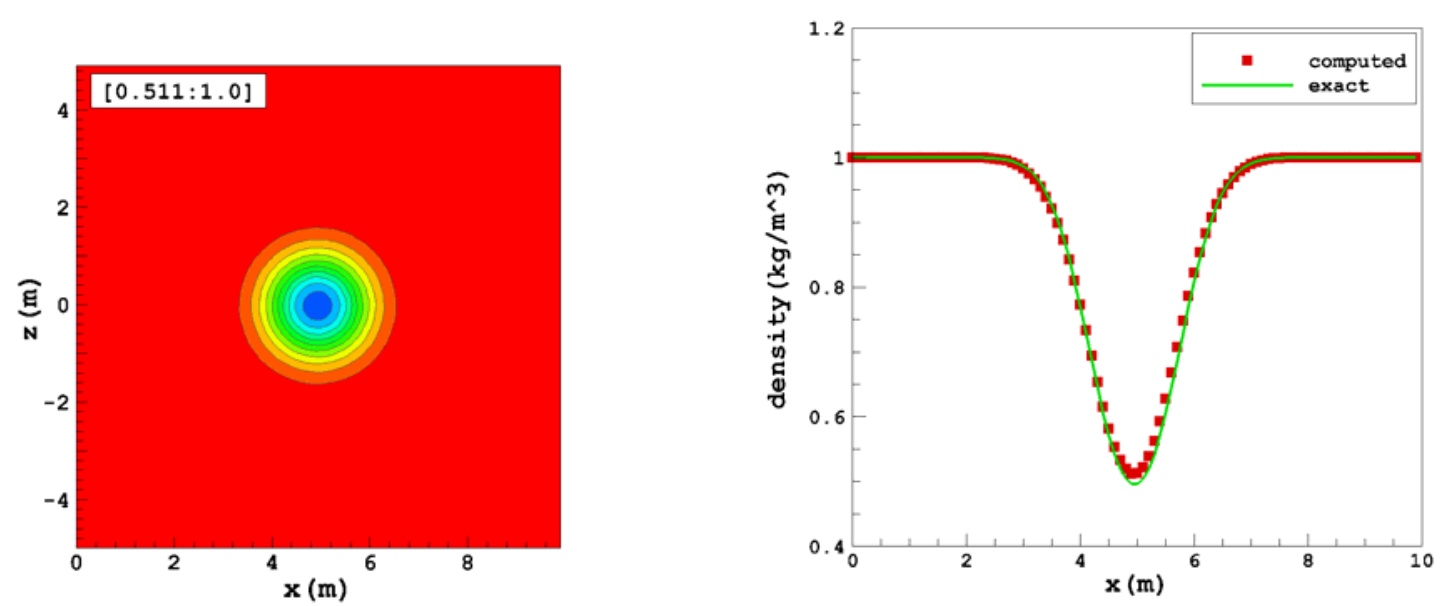

Figure 2. Moving Vortex. Computed density $\left(\mathrm{kg} / \mathrm{m}^{3}\right)$ at time $=100 \mathrm{~s}$ (left) and the comparison of the computed solution with the analytical solution at $z=0$ and time $=100$ s (right). Mesh size $=100 \times 100$ cells.
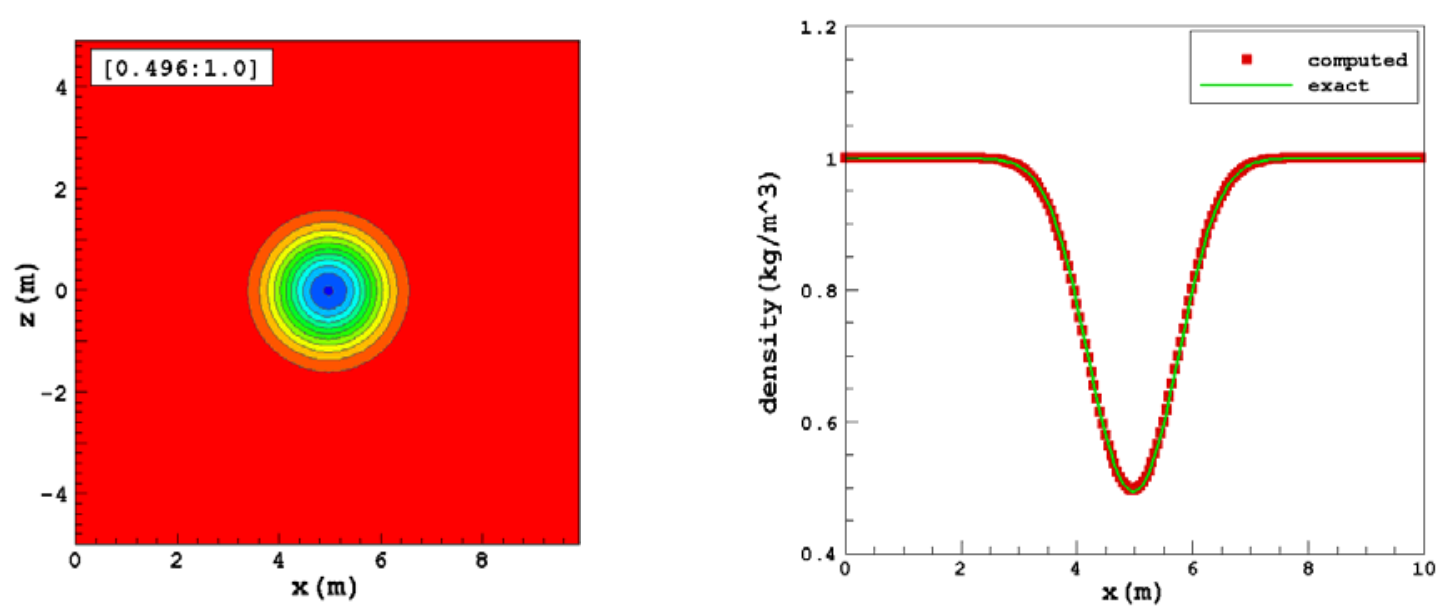

Figure 3. Moving Vortex. Computed density $\left(\mathrm{kg} / \mathrm{m}^{3}\right)$ at time $=100 \mathrm{~s}$ (left) and the comparison of the computed solution with the analytical solution at $z=0$ and time $=100$ s (right). Mesh $\operatorname{size}=200 \times 200$ cells. 


\section{B. Transport of Smooth Density Distribution}

Deiterding (2000) suggested a simple test case with an analytical solution for evaluating numerical schemes for the Euler equations with a gravitational source term. The Euler equations can be reduced to a single transport equation for density:

$$
\begin{aligned}
& u(x, z, t)=u_{0} \\
& w(x, z, t)=w_{0}-g t \\
& p(x, z, t)=p_{0}
\end{aligned}
$$

The following relation describes the advection of an initial arbitrary density profile (Deiterding 2000):

$$
\rho(x, z, t)=\rho_{0}\left(x-u_{0} t, z-w_{0} t+\frac{1}{2} g t^{2}\right)
$$

The computational domain in this case was defined as $(x, z) \in[0,2] \times[0,2] m$ with $t \in[0,0.5] \mathrm{s}$. Periodic boundary conditions were used in all directions. The transport of density distribution is given by:

$$
\begin{aligned}
& x_{c}(t)=x_{0}+u_{0} t \\
& z_{c}(t)=z_{0}+w_{0} t-\frac{1}{2} g t^{2}
\end{aligned}
$$

The initial density distribution is defined as follows:

$$
\rho(r)= \begin{cases}\rho_{1}+\rho_{2}\left[\sin \left(\frac{r}{R} \pi+\frac{\pi}{2}\right)+1\right] & \text { if } 0 \leq r<R \\ \rho_{1} & \text { if } R \leq r\end{cases}
$$

where,

$$
r(x, z, t)=\sqrt{\left(x-x_{c}(t)\right)^{2}+\left(z-z_{c}(t)\right)^{2}}
$$

In the above equations $\rho_{1}=1 \mathrm{~kg} / \mathrm{m}^{3}, \rho_{2}=0.05 \mathrm{~kg} / \mathrm{m}^{3}, x_{0}=0.75 \mathrm{~m}, \mathrm{z}_{0}=0.75 \mathrm{~m}, u_{0}=1 \mathrm{~m} / \mathrm{s}, w_{0}=1.25 \mathrm{~m} / \mathrm{s}, p_{0}=1 \mathrm{~Pa}, g$ $=1 \mathrm{~m} / \mathrm{s}^{2}$, and $\gamma=1.39$.

The simulation was run for different mesh resolutions and the root mean square errors in the density $\left(E_{\rho}\right)$ and the velocity $\left(E_{\mathrm{v}}\right)$ fields were calculated for each simulation (Table 2).

The density distribution at time $=0.5 \mathrm{~s}$ is shown in Figure 4 . The computed density profile along the mesh diagonal is also compared with the exact analytical solution in Figure 4. The computed results are in excellent agreement with the exact analytical solution.

Table 2: Transport of Smooth Density Distribution

\begin{tabular}{|c|c|c|c|}
\hline$\#$ of cells & $\Delta \mathbf{x}(\mathbf{m})$ & $\boldsymbol{E}_{\boldsymbol{\rho}}$ & $\boldsymbol{E}_{\mathbf{v}}$ \\
\hline $40 \times 40$ & 0.05 & $0.536237 \mathrm{E}-03$ & $0.289277 \mathrm{E}-15$ \\
\hline $80 \times 80$ & 0.025 & $0.210343 \mathrm{E}-03$ & $0.361827 \mathrm{E}-15$ \\
\hline $160 \times 160$ & 0.0125 & $0.940737 \mathrm{E}-04$ & $0.362340 \mathrm{E}-15$ \\
\hline $320 \times 320$ & 0.00625 & $0.449019 \mathrm{E}-04$ & $0.776312 \mathrm{E}-15$ \\
\hline
\end{tabular}



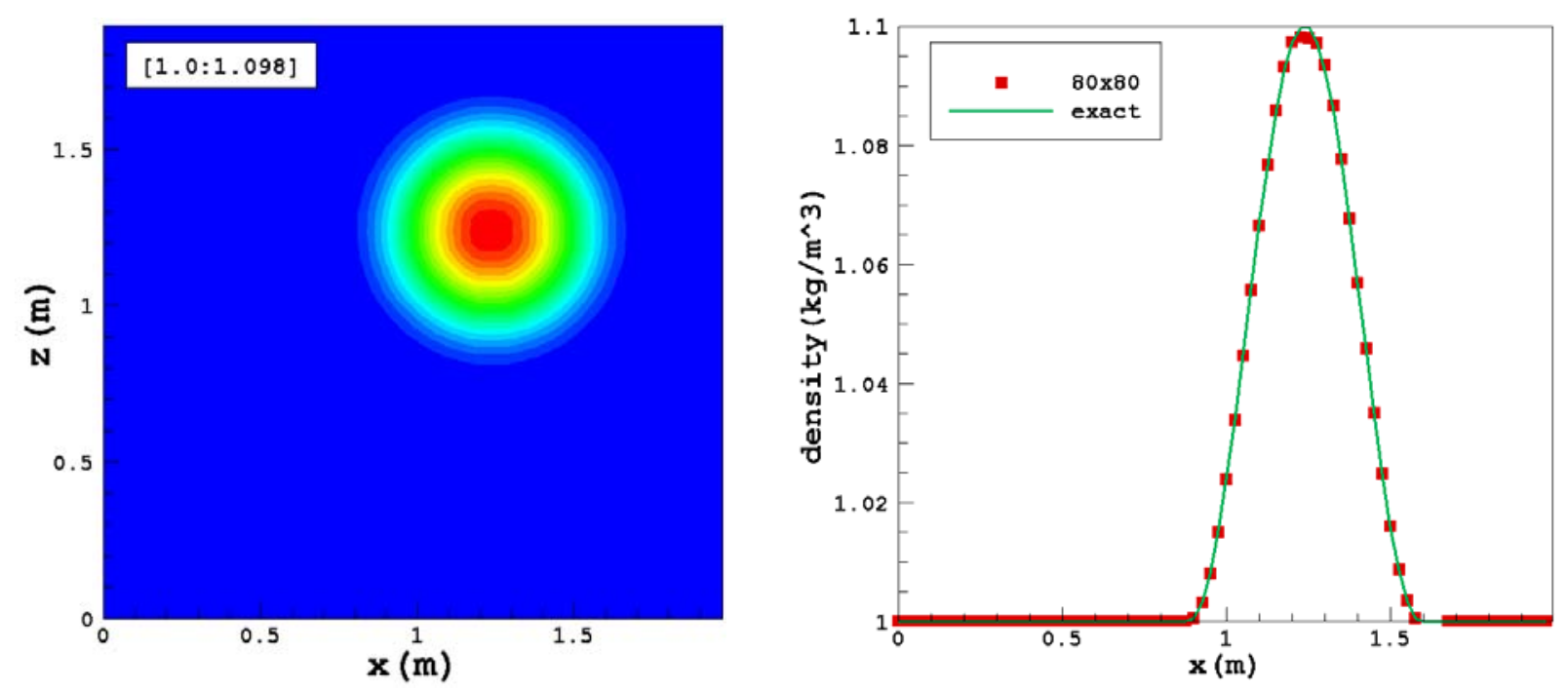

Figure 4. Transport of Density Distribution. The computed density distribution $\left(\mathrm{kg} / \mathrm{m}^{3}\right)$ at time $=0.5 \mathrm{~s}$ is shown in the left panel and the comparison of the computed solution with the analytical solution along the mesh diagonal at time $=0.5 \mathrm{~s}$ is shown in the right panel.

\section{Rayleigh-Taylor Instability}

Simulation of the Rayleigh-Taylor instability has been described in several publications (Almgren et al. 2010; Liska and Wendroff 2003; $\mathrm{Li}$ et al. 1996) and is often used as a validation test case. The computational domain in this case was defined by $(x, z) \in[0,0.5] \times[0,1] \mathrm{m}$ with $t \in[0,2.5] \mathrm{s}$. The mesh size was $256 \times 512$ cells. Periodic boundary conditions were used in the lateral and the top and bottom boundaries were set to solid walls. The initial $u$-velocity and the $w$-velocity were set to zero. The density was set to $\rho_{1}=1 \mathrm{~kg} / \mathrm{m}^{3}$ in the lower half of the domain and $\rho_{2}=2 \mathrm{~kg} / \mathrm{m}^{3}$ in the upper half of the domain. Pressure was initialized using the hydrostatic equation:

$$
p(z)=\left\{\begin{array}{cc}
p_{0}-\rho_{1} g z & z<L_{z} / 2 \\
p_{0}-\rho_{1} g L_{z} / 2-\rho_{2} g\left(z-L_{z} / 2\right) & z>L_{z} / 2
\end{array}\right.
$$

where $p_{0}$ is the reference pressure and was set to $5 P a$. The acceleration due to gravity, $g$ was set to $1 \mathrm{~m} / \mathrm{s}^{2} . L_{z}=1 \mathrm{~m}$ is the height of the computational domain. A single-mode perturbation was introduced in density at the interface of heavier and lighter fluids:

$$
\rho(x, z)=\rho_{1}+\frac{\rho_{2}-\rho_{1}}{2}\left[1+\tanh \left(\frac{z-\psi(x)}{0.005}\right)\right]
$$

where,

$$
\psi(x)=\frac{L_{z}}{2}+0.01 \frac{\cos (4 \pi x)+\cos \left(4 \pi\left(L_{x}-x\right)\right)}{2}
$$

In Eq. (25), $L_{\mathrm{x}}=0.5 \mathrm{~m}$ is the width of the computational domain. The tanh function in Eq. (24) is used to smooth the density profile at the interface (Almgren et al. 2010). The initial conditions and the evolution of the instability are shown in Figure 5. The scheme was able to capture the onset of the instability accurately and the solution is in good agreement with previously reported results in the literature (e.g., Almgren et al. 2010). 

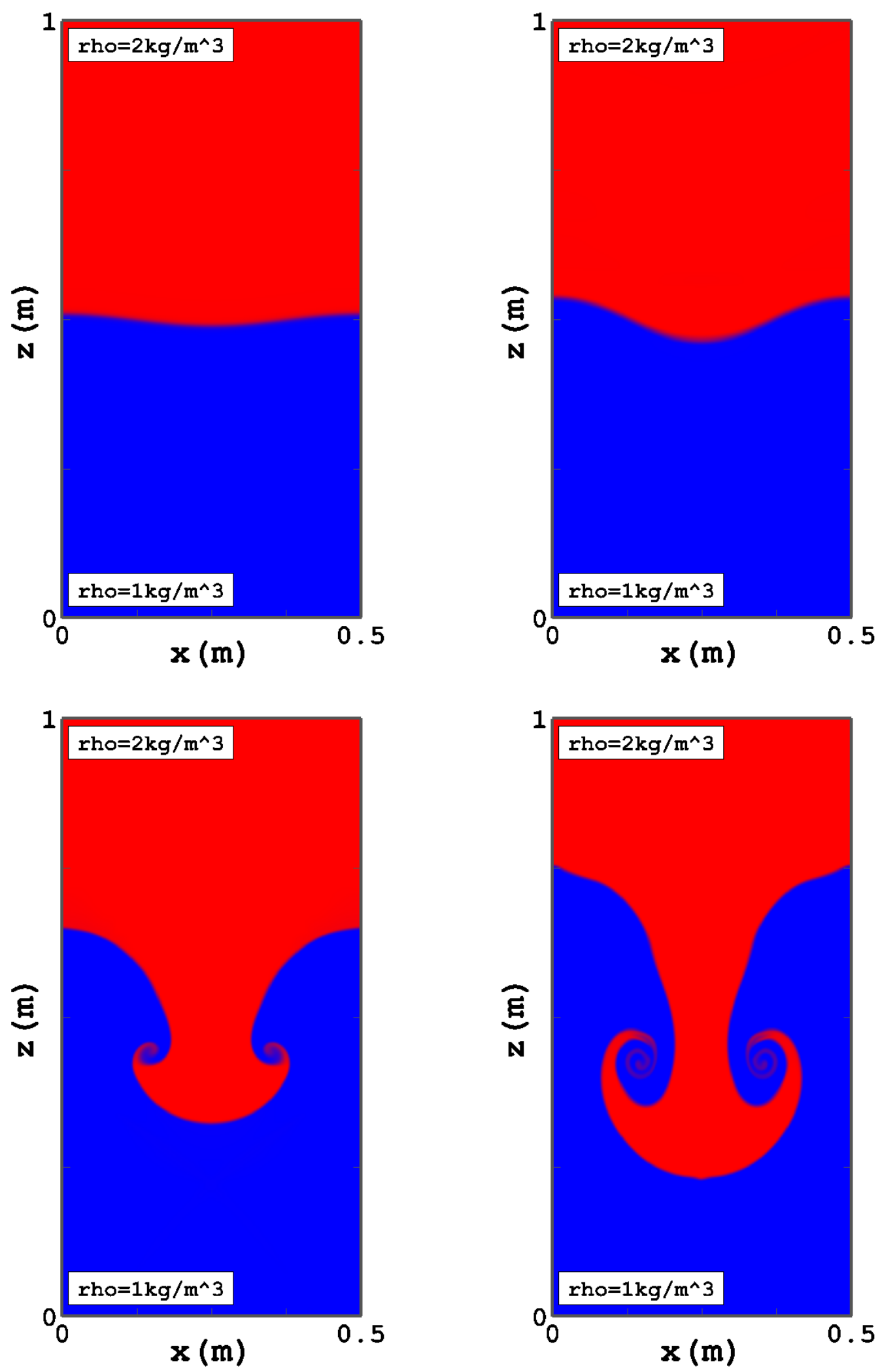

Figure 5. Rayleigh-Taylor Instability. The initial density field (top left panel); density field at $1 s$ (top right panel); at $2 s$ (bottom left panel); and at 2.5s (bottom right panel). Mesh size = 256 x 512 cells. 


\section{Non-hydrostatic Inertia-Gravity Waves}

The simulation of inertia-gravity waves (Skamarock and Klemp 1994) on the non-hydrostatic scale is described in this section. The computational domain was defined by $(x, z) \in[0,300] \times[0,10] \mathrm{km}$ with $t \in[0,3000] \mathrm{s}$. The mesh had a resolution of $1 \mathrm{~km}$ in the $x$ and $50 \mathrm{~m}$ in the $z$-direction $(300 \times 200$ cells). Periodic boundary conditions were used in the lateral and the top and bottom boundaries were set to solid walls. The domain was initialized by a constant Brunt-Väisälä frequency, $N=10^{-2} s^{-1}$. The waves were excited by an initial potential temperature perturbation given by:

$$
\theta(x, z, 0)=\Delta \theta_{0} \frac{\sin (\pi z / H)}{1+\left(x-x_{c}\right)^{2} / a^{2}}
$$

The amplitude of the initial potential temperature perturbation, $\Delta \theta_{0}$ was set to $10^{-2} \mathrm{~K}$. The height $H$ of the domain was $10 \mathrm{~km}$, the perturbation half width was $a=5 \mathrm{~km}$. The perturbation was initialized at $x_{c}=L_{\mathrm{x}} / 3$, where, $L_{\mathrm{x}}$ is the width of the domain $(300 \mathrm{~km})$. A uniform $u$-velocity of $20 \mathrm{~m} / \mathrm{s}$ was imposed in the domain and the $w$-velocity was set to zero. The initial potential temperature perturbation field is shown in Figure 6 and Figure 7 shows the computed potential temperature perturbation at time $=3000 \mathrm{~s}$. Overall, the solution agrees well with other results reported in literature (Ahmad and Lindeman 2007; Giraldo and Restelli 2008). Compared to Giraldo and Restelli (2008) who used a higher-order Discontinuous Galerkin Method, the results are slightly diffusive (the wave in the middle of the domain has a lower peak). The final minimum and maximum potential temperature perturbation in Giraldo and Restelli (2008) are $\theta^{\prime} \in\left[-1.51 \times 10^{-3}, 2.78 \times 10^{-3}\right] K$ compared to $\theta^{\prime} \in\left[-1.41 \times 10^{-3}, 2.83 \times 10^{-3}\right] K$ in the current study.

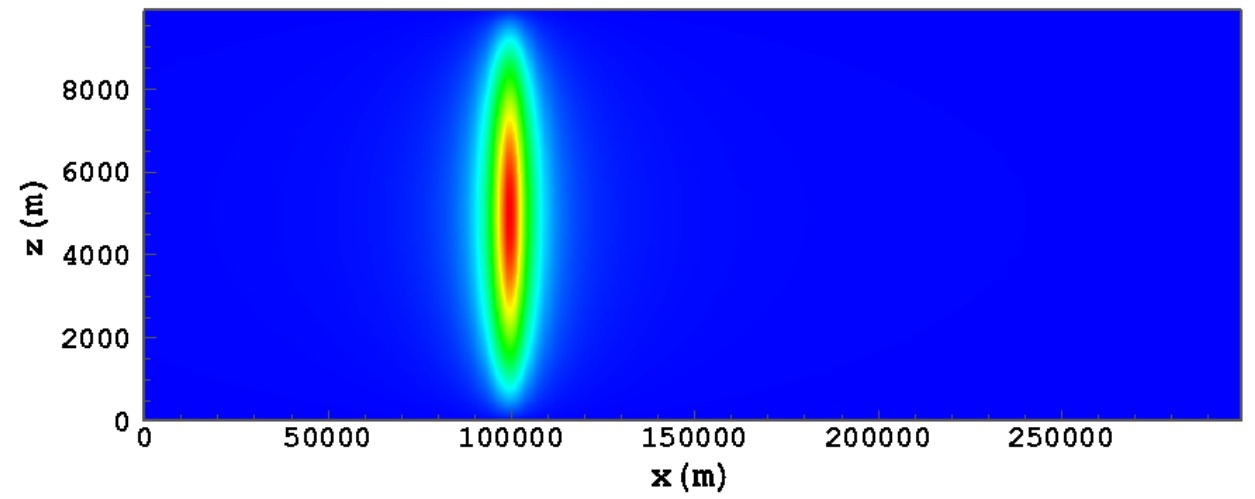

Figure 6. Inertia Gravity Waves. The initial potential perturbation field $(K)$ is shown in the figure. The field minimum $=0 \mathrm{~K}$ and the maximum $=0.01 \mathrm{~K}$. Mesh size $=300 \times 200$ cells .

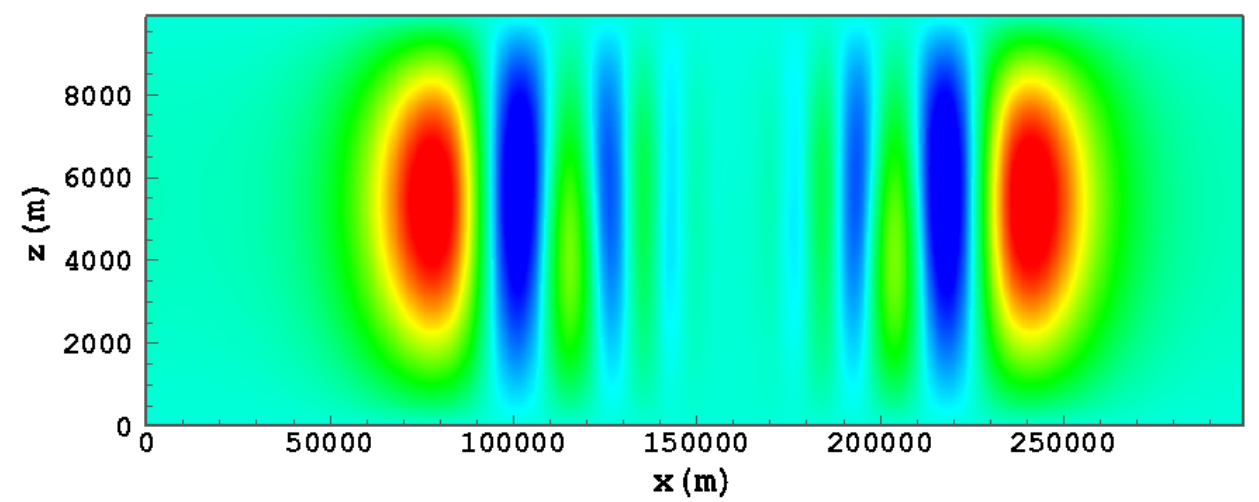

Figure 7. Inertia Gravity Waves. The potential temperature perturbation field $(K)$ at time $=3000$ s. The field minimum $=-0.00141 \mathrm{~K}$ and the maximum $=0.00283 \mathrm{~K}$. Mesh size $=300 \times 200$ cells. 


\section{E. Density Current}

The simulation of density current (Straka et al. 1993; Ahmad and Lindeman 2007; Giraldo and Restelli 2008) is described in this section. The computational domain was defined by $(x, z) \in[0,25] \times[0,6.4] \mathrm{km}$ with $t \in[0,900] \mathrm{s}$. The mesh had a uniform resolution of $50 \mathrm{~m}$ in both $x$ and $z$-directions $(500 \times 128$ cells). Farfield/outflow boundary conditions were used for the right lateral boundary and all other boundaries were set to solid walls. The domain was initialized for a neutral atmosphere by setting the potential temperature at $300 \mathrm{~K}$. The initial $u$-velocity and the $w$ velocity were set to zero. A vertical temperature profile was defined for the entire domain using the following relation:

$$
T=300-\frac{z g}{c_{p}}
$$

A cold bubble was initialized by adding a perturbation in the temperature field using the following relation:

$$
\Delta T=\left\{\begin{array}{lr}
0.0 & \text { if } L>1.0, \\
-15.0\left[\frac{\cos (\pi L)+1.0}{2}\right] & \text { if } L \leq 1.0
\end{array}\right.
$$

where,

$$
L=\sqrt{\left(\frac{x-x_{c}}{x_{r}}\right)^{2}+\left(\frac{z-z_{c}}{z_{r}}\right)^{2}}
$$

where, $\left(x_{c}, z_{c}\right)=(0,3) \mathrm{km}$ and $\left(x_{r}, z_{r}\right)=(4,2) \mathrm{km}$. The initial potential temperature perturbation field is shown in Figure 8. A constant viscosity $\left(\mu=75 \mathrm{~m}^{2} / \mathrm{s}\right)$ was used in the calculation of momentum and energy viscous fluxes. The Prandtl number was set to 0.76 .

This test case can be considered as an idealized downburst gust front. As the cold air descends due to negative buoyancy, strong downdrafts develop at the center of the cold bubble. When the cold air reaches the ground, it is rolled up and a front is formed. As this front propagates, shear is generated at the top boundary of the front. The benchmark solution (Straka et al. 1993) consists of three rotors, which develop at the top boundary of the front due to Kelvin-Helmholtz type instability. The potential temperature perturbation field at time $=900 \mathrm{~s}$ is given in Figure 9, which shows the three characteristic rotors of the Straka benchmark. The front is located at $x=15326 \mathrm{~m}$. The location of the front in the Straka reference solution is at $x=15537 \mathrm{~m}$. The location of front in Giraldo and Restelli (2008) is at $x=14789 \mathrm{~m}$. The reference solution in Straka et al. (1993) and Giraldo and Restelli (2008) are on a 25m resolution mesh whereas a mesh resolution of $50 \mathrm{~m}$ was used in the current study. The present results also compare well with other reported solutions of the benchmark (e.g., Janjić et al. 2001; Xue et al. 2000).

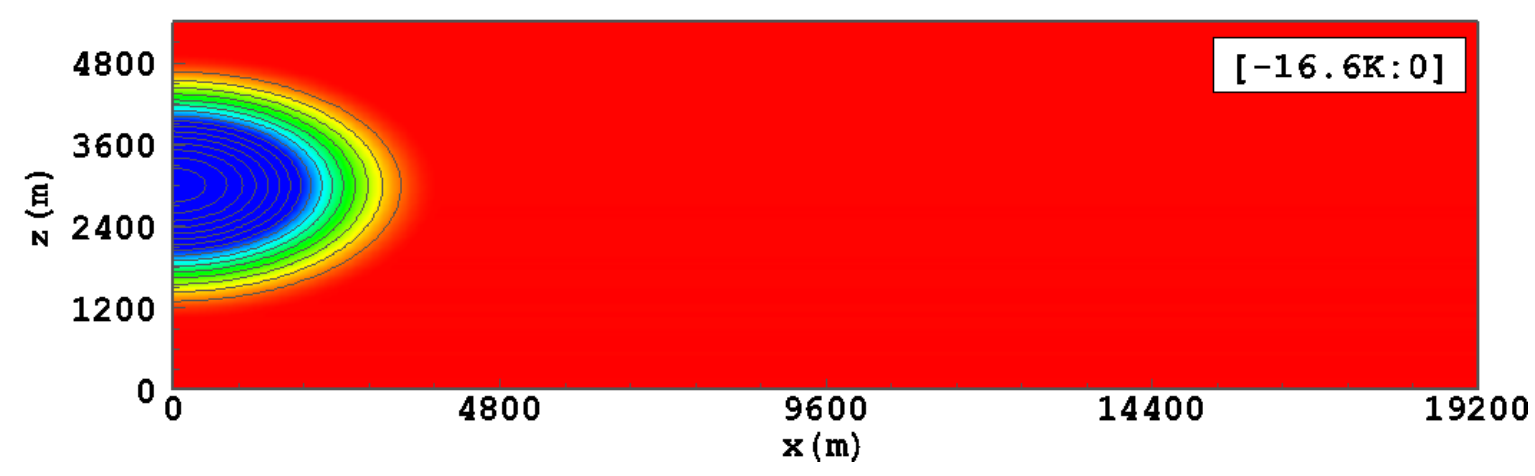

Figure 8. Density Current. The initial potential perturbation field $(K)$ is shown in the figure. The field minimum $=-16.6 \mathrm{~K}$ and the maximum $=0 \mathrm{~K}$. Mesh size $=500 \times 128$ cells. 


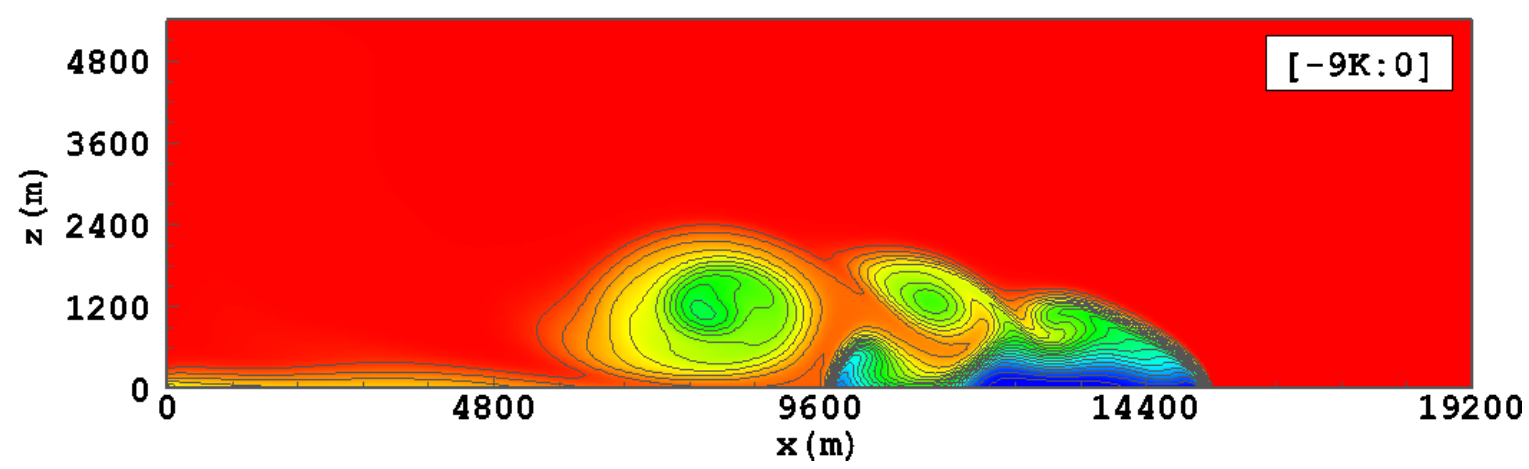

Figure 9. Density Current. The potential temperature perturbation field $(K)$ at time $=900 s$. The contour values shown in the figure are from $-9 K$ to $0 K$. The Mesh size $=500 \times 128$ cells.

\section{Summary}

LeVeque's high-resolution wave-propagation method was applied to non-hydrostatic atmospheric flows on meso- and micro-scales. The equation for total energy conservation was used instead of potential temperature conservation in the simulations. The results show that the numerical method is fully conservative and is able to discretize the source due to gravity without introducing errors in the solution. Comparisons with exact analytical solutions show that the flow solver has relatively small diffusion and dispersion errors. In the case of isentropic vortex and density transport case, the computed solutions are in excellent agreement with the exact analytical solutions. The simulations of the Rayleigh-Taylor instability, nonhydrostatic inertia gravity waves, and the density current also compare well with previously reported results in the literature. Since the eigenstructure of the Euler equations is well known, the scheme can be extended to three dimensions for simulating realistic flows. It is especially well-suited for simulating atmospheric flows in which sharp gradients of temperature, potential temperature and winds can develop (e.g., fronts, supercell thunderstorms, tornadoes).

\section{Acknowledgment}

This work is sponsored under NASA's Concepts \& Technology Development Project of the Airspace Systems Program.

\section{References}

Ahmad, N., D. Bacon, A. Sarma, D. Koračin, R. Vellore, Z. Boybeyi, and J. Lindeman, "Simulations of Non-hydrostatic Atmosphere using Conservation Laws Package", AIAA Paper 2007-84.

Ahmad, N., and J. Lindeman, "Euler Solutions using Flux-based Wave Decomposition". International Journal for Numerical Methods in Fluids, Vol. 54:1, 2007, pp. 41-72.

Almgren, A. S., V. E. Beckner, J. B. Bell, M. S. Day, L. H. Howell, C. C. Joggerst, M. J. Lijewski, A. Nonaka, M. Singer, M. Zingale, "CASTRO: A New Compressible Astrophysical Solver. I. Hydrodynamics and Self-Gravity", The Astrophysical Journal, Vol. 715, 2010, pp. 1221-1238.

Bacon, D. P., N. N. Ahmad, Z. Boybeyi, T. J. Dunn, M. S. Hall, P. C. S. Lee, R. A. Sarma, M. D. Turner, K. Waight, S. Young, and J. Zack, “A Dynamically Adapting Weather and Dispersion Model: The Operational Multiscale Environment Model with Grid Adaptivity (OMEGA)", Monthly Weather Review, Vol. 128, 2000, pp. 2044-2076.

Bale, D., R. J. LeVeque, S. Mitran, and J. A. Rossmanith, “A wave-propagation method for conservation laws and balance laws with spatially varying flux functions”, SIAM Journal of Scientific Computing, Vol. 26, 1908, pp. 177-183.

Botta, N., R. Klein, S. Langenberg, and S. Lutzenkirchen, "Well balanced finite volume methods for nearly hydrostatic flows”, Journal of Computational Physics, Vol. 196, 2004, pp. 539-565.

Collela, P., and P. R. Woodward, “The Piecewise Parabolic Method (PPM) for Gas-Dynamical Simulations", Journal of Computational Physics, Vol. 54, 1984, pp. 174-201. 
Das, P., “A Non-Archimedean Approach to the Equations of Convection Dynamics”, Journal of Atmospheric Sciences, Vol. 36, 1979, pp. 2183-2190.

Deiterding, R., "Two-dimensional Euler equations with gravity”. Technical Report NMWR-00-1. Institute of Mathematics, Technical University Cottbus, Germany. 2000.

Giraldo, F. X., and M. Restelli, “A study of spectral element and discontinuous Galerkin methods for the Navier-Stokes equations in nonhydrostatic mesoscale atmospheric modeling: Equations sets and test cases", Journal of Computational Physics, Vol. 227, 2008, pp. 3849-3877.

Godunov, S. K., “A Finite Difference Method for the Computation of Discontinuous Solutions of the Equations of Fluid Dynamics", Matematicheskii Sbornik, Vol. 47, 1959, pp. 357-393.

Hodur, R. M., "The Naval Research Laboratory’s Couples Ocean/Atmosphere Mesoscale Prediction System (COAMPS)”, Monthly Weather Review, Vol. 125, pp. 1414-1430.

Janjić, Z. I., J. P. Gerrity, and S. Nickovic, “An Alternative Approach to Nonhydrostatic Modeling”, Monthly Weather Review, Vol. 129, 2001, pp. 1164-1178.

LeVeque, R. J., "High-resolution conservative algorithms for advection in incompressible flow", SIAM Journal of Numerical Analysis, Vol. 33, 1996, pp. 627-665.

LeVeque, R. J., "Wave propagation algorithms for multi-dimensional hyperbolic systems", Journal of Computational Physics, Vol. 131, 1997, pp. 327-353.

LeVeque, R. J., "Finite Volume Methods for Hyperbolic Problems”, Cambridge University Press, 2002.

Li, X. L., B. X. Jin, J. Glimm, "Numerical Study for the Three-Dimensional Rayleigh-Taylor Instability through the TVD/AC Scheme and Parallel Computation”, Journal of Computational Physics, Vol. 126, 1996, pp. 343-355.

Liska, R., B. Wendroff, "Comparison of Several Difference Schemes on 1D and 2D Test Problems for the Euler Equations”, SIAM Journal of Scientific Computing, Vol. 25, 2003, pp. 995-1017.

Pielke, R., W. R. Cotton, R. L. Walko, C. J. Tremback, W. A. Lyons, L. D. Grasso, M. E. Nicholls, “A Comprehensive Meteorological Modeling System - RAMS”, Meteorology and Atmospheric Physics, Vol. 49, 1992, pp. 69-91.

Proctor, F. H., “The Terminal Area Simulation System / Volume 1: Theoretical Formulation”, NASA Report 4046. 1987.

Skamarock, W. C., and J. B. Klemp, "Efficiency and Accuracy of the Klemp-Wilhelmson Time-Splitting Technique", Monthly Weather Review, Vol. 122, 1994, pp. 2623-2630.

Straka, J. M., R. B. Wilhelmson, L. J. Wicker, J. R. Anderson, and K. K. Droegemeier, "Numerical Solutions of a Non-linear Density Current: A Benchmark Solution and Comparisons”, International Journal for Numerical Methods in Fluids, Vol. 17, 1993, pp. 1-22.

Xue, M., K. K. Droegemeier, and V. Wong, "The Advanced Regional Prediction System (ARPS) - A multi-scale nonhydrostatic atmospheric simulation and prediction model. Part I: Model dynamics and verification", Meteorology and Atmospheric Physics, Vol. 75, 2000, pp. 161-193.

Yee, H. C., N. D. Sandham, M. J. Djomehri "Low-Dissipative High-Order Shock-Capturing Methods Using CharacteristicBased Filters", Journal of Computational Physics, Vol. 150, 1999, pp. 199-238. 